\title{
Dependence of the vacuum energy on spherically symmetric background fields
}

\author{
Michael Bordag*, Meik Hellmund \\ Universität Leipzig, Institut für Theoretische Physik, \\ Augustusplatz 10, 04109 Leipzig, Germany \\ Klaus Kirsten; \\ The University of Manchester, Department of Theoretical Physics \\ Manchester, England \\ March 4, 2018

\begin{abstract}
The vacuum energy of a scalar field in a spherically symmetric background field is considered. Based on previous work [Phys. Rev. D 53 (1996) 5753], the numerical procedure is refined further and applied to several examples. We provide numerical evidence that repulsive potentials lead to positive contributions to the vacuum energy. Furthermore, the crucial role played by bound-states is clearly seen.
\end{abstract}

Pacs number(s): 11.10-z; 02.30.-f; 11.10Gh

\section{Introduction}

The calculation of vacuum energies in the context of quantum field theory under external conditions plays an important role in several areas of modern theoretical physics. External conditions present may be classical fields (for example gravitational [1] and electromagnetic fields, monopoles [2, 3], Sphalerons [4] or electroweak Skyrmions [5]), or they may be realized through boundaries at which the quantum field has to satisfy certain boundary conditions (see for example

\footnotetext{
${ }^{*}$ E-mail: Michael.Bordag@itp.uni-leipzig.de

${ }^{\dagger}$ E-mail: Meik.Hellmund@itp.uni-leipzig.de

${ }_{\ddagger}^{\ddagger}$ E-mail: klaus@a13.ph.man.ac.uk
} 
[6]). External fields most naturally appear as classical solutions of some physical nonlinear equations, but they may also be viewed as a localized property of space representing a distribution of matter within which the quantum field exists. For external fields realized as boundaries this situation is usually called Casimir effect. There is a number of generalizations towards weakened boundaries, e.g., by introducing semi hard walls [7] or transparent boundaries realized by delta functions [8]. For actual interest in such calculations see, e.g., [9, 10].

A formalism how to deal with arbitrary background fields, which we use here, has been developed in [11] for a background depending on one cartesian coordinate and in [12] for a general spherically symmetric background. However, these methods had been applied to a square well potential only where the interesting quantities can be expressed in terms of Bessel functions.

The aim of the present work is to show that this formalism is well suited for arbitrarily shaped spherically symmetric background fields. We investigate in detail different types of background fields as examples. It is observed that the main feature of the vacuum energy is determined by the number (and depth) of bound states. As long as these are kept fixed the general behavior of its energy when varying parameters is the same within the class of examples considered. At the same time we find that the presence of bound states does not fix the sign of the ground state energy.

Similar calculations, however with a less extend of details and using other techniques, have been performed earlier, e.g., in [13] and [14.

The paper is organized as follows. In the next section the basic notations of the method used are explained. In the third section the 'asymptotic' contribution is calculated. In the fourth section the numerical procedure is developed in detail followed by a section containing the examples calculated. The results are discussed in the conclusions.

\section{The model, its renormalization and some ba- sic results}

We consider the theory described by the Lagrangian

$$
L=\frac{1}{2} \Phi\left(\square-M^{2}-\lambda \Phi^{2}\right) \Phi+\frac{1}{2} \varphi\left(\square-m^{2}-\lambda^{\prime} \Phi^{2}\right) \varphi .
$$

Here the field $\Phi$ is a classical background field. By means of

$$
V(x)=\lambda^{\prime} \Phi^{2}
$$

it defines the potential in (1) for the field $\varphi(x)$, which should be quantized in the background of $V(x)$. 
Our interest is in the vacuum energy of the theory which is defined as half the sum of the one particle energies of the field $\varphi(x)$

$$
E_{\varphi}[\Phi]=\frac{1}{2} \sum_{(n)}\left(\lambda_{(n)}^{2}+m^{2}\right)^{1 / 2-s} \mu^{2 s} .
$$

Here $s$ is the regularization parameter with $s \rightarrow 0$ in the end and $\mu$ is an arbitrary mass parameter. The $\lambda_{(n)}^{2}$ are the one-particle energy eigenvalues determined through

$$
(-\Delta+V(x)) \phi_{(n)}(x)=\lambda_{(n)}^{2} \phi_{(n)}(x) .
$$

For the moment a finite volume is assumed to have discrete eigenvalues.

The spectral function appearing in Eq. (3) is related to the zeta function of the wave operator (四),

$$
\zeta_{V}(s)=\sum_{(n)}\left(\lambda_{(n)}^{2}+m^{2}\right)^{-s}
$$

by

$$
E_{\varphi}[\Phi]=\frac{1}{2} \zeta_{V}(s-1 / 2) \mu^{2 s}
$$

The ultraviolet divergences contained in the ground state energy (3) needs to be renormalized. The divergences are known and best expressed in terms of the heat kernel coefficients related to the operator defined in Eq. (四). Then the divergent part of the ground state energy reads

$$
\begin{aligned}
E_{\varphi}^{d i v}[\Phi]= & -\frac{m^{4}}{64 \pi^{2}}\left(\frac{1}{s}+\ln \frac{4 \mu^{2}}{m^{2}}-\frac{1}{2}\right) A_{0} \\
& +\frac{m^{2}}{32 \pi^{2}}\left(\frac{1}{s}+\ln \frac{4 \mu^{2}}{m^{2}}-1\right) A_{1} \\
& -\frac{1}{32 \pi^{2}}\left(\frac{1}{s}+\ln \frac{4 \mu^{2}}{m^{2}}-2\right) A_{2},
\end{aligned}
$$

with the coefficients $A_{1}=-\int \mathrm{d} \vec{x} V(x), A_{2}=\frac{1}{2} \int \mathrm{d} \vec{x} V(x)^{2}$ and $A_{0}$ is the spatial volume. The contribution of $A_{0}$, because it does not depend on the background will be dropped from here on without further comment.

The renormalization procedure is set by the definition

$$
E_{\varphi}^{r e n}=E_{\varphi}[\Phi]-E_{\varphi}^{d i v}[\Phi]
$$

and the corresponding counter terms can be absorbed into a redefinition of the parameters $M^{2}$ and $\lambda$ of the classical background field whose energy reads

$$
E_{\text {class }}[\Phi]=\frac{1}{2} \int \mathrm{d} \vec{x}(\nabla \Phi)^{2}-\frac{M^{2}}{2 \lambda^{\prime}} A_{1}+\frac{\lambda}{\lambda^{\prime 2}} A_{2} .
$$


It might be useful to note that among the divergent contributions (7) to the ground state energy there are no terms proportional to the kinetic part in the Lagrangian of the background $\Phi(x)$. Within the given framework this is a simple consequence of the structure of the heat kernel coefficients. Sometimes for the divergent part of the ground state energy the first 2 terms of the perturbative expansion of $E_{0}[\Phi]$ with respect to powers of the background field $\Phi$ is taken (e.g., in [14]). This has the disadvantage of containing nonlocal contributions (in opposite to the heat kernel expansion expansion which is local) and invites to discuss the kinetic term among the divergent ones which is in fact unnecessary.

As the renormalization procedure is not unique, a normalization condition must be imposed. We require

$$
\lim _{m \rightarrow \infty} E_{\varphi}^{r e n}[\Phi]=0
$$

For a massive field this is the natural condition requiring the energy of the quantum fluctuation to vanish for the field becoming heavy. As discussed in [15] this delivers a unique definition of the ground state energy (in opposite to the case of a massless field, see the discussion in [15 and also in [16]).

To proceed we follow [12] and use the representation of the regularized ground state energy in terms of the Jost function $f_{l}(k)$ of the scattering problem corresponding to (4)

$$
E_{\varphi}[\Phi]=-\frac{\cos \pi s}{\pi} \mu^{2 s} \sum_{l=0}^{\infty}(l+1 / 2) \int_{m}^{\infty} d k\left[k^{2}-m^{2}\right]^{\frac{1}{2}-s} \frac{\partial}{\partial k} \ln f_{l}(i k) .
$$

We remind the definition of the so-called regular solution $\phi_{n, l}(r)$ of the radial wave equation (see, e.g., [17])

$$
\left[\frac{d^{2}}{d r^{2}}-\frac{l(l+1)}{r^{2}}-V(r)+k^{2}\right] \phi_{n, l}(r)=0 .
$$

It is the solution, which for $r \rightarrow 0$ behaves as the free (i.e., without potential) solution. The Jost function (and its complex conjugate) are defined as the coefficients in the asymptotic expansion of this solution as $r \rightarrow \infty$ :

$$
\phi_{l, p}(r) \underset{r \rightarrow \infty}{\sim} \frac{i}{2}\left[f_{l}(p) \hat{h}_{l}^{-}(p r)-f_{l}^{*}(p) \hat{h}_{l}^{+}(p r)\right],
$$

where $\hat{h}_{l}^{-}(p r)$ and $\hat{h}_{l}^{+}(p r)$ are the Riccati-Hankel functions.

Instead of dealing explicitly with eigenvalues, the vacuum energy is expressed in terms of the Jost function. Although representation (11) does not reveal explicitly the dependence of the energy on the bound states, in 12 it was shown that their contribution is correctly included. This, and the non oscillating behavior of 
the Jost function on the imaginary axis (cf. (111) gives this representation calculational advantages with respect to alternative representation (e.g. integrating the scattering phases over the real $k$-axis).

To proceed we have to perform the analytic continuation in $s$ to $s=0$ in representation (8) of $E_{\varphi}^{\mathrm{ren}}$ using (11) for $E_{\varphi}[\Phi]$. Although we known that the result is finite, this task requires some work. The point is that one cannot put $s=0$ under the sign of the sum and the integral in (11) being inserted into Eq. (8). The reason is the lack of convergence for $k$ and $l$ large. Therefor we use the uniform asymptotic expansion $\ln f_{k}^{\text {asym }}(i k)$ of the logarithm of the Jost function for both, $k$ and $l$ large. We subtract and add it to the integrand. As a result we get the sum of two parts

$$
E_{\varphi}^{\mathrm{ren}}[\Phi]=E_{f}[\Phi]+E_{a s}[\Phi]
$$

with

$$
E_{f}[\Phi]=-\frac{1}{\pi} \sum_{l=0}^{\infty}(l+1 / 2) \int_{m}^{\infty} d k\left[k^{2}-m^{2}\right]^{\frac{1}{2}} \frac{\partial}{\partial k}\left[\ln f_{l}(i k)-\ln f_{l}^{a s y m}(i k)\right]
$$

and

$E_{a s}[\Phi]=-\frac{\cos (\pi s)}{\pi} \mu^{2 s} \sum_{l=0}^{\infty}(l+1 / 2) \int_{m}^{\infty} d k\left[k^{2}-m^{2}\right]^{\frac{1}{2}-s} \frac{\partial}{\partial k} \ln f_{l}^{a s y m}(i k)-E_{\varphi}^{\mathrm{div}}$.

The first contribution, $E_{f}[\Phi]$ is called the finite part. There the regularization parameter $s$ has been put to zero directly because the sum and the integral are convergent due to the subtraction. The second contribution, $E_{a s}[\Phi]$, is called the asymptotic contribution. It has, as we know, a finite limit for $s \rightarrow 0$ which will be determined below. The asymptotic expansion $\ln f_{l}^{a s y m}(i k)$ for $\nu \rightarrow \infty, k \rightarrow \infty$, $\nu / k$ fixed, was determined in [12] up to the required order of $\nu^{-3}$. It reads

$$
\begin{aligned}
\ln f_{l}^{\text {asym }}(i k)= & \frac{1}{2 \nu} \int_{0}^{\infty} d r \frac{r V(r)}{\left[1+\left(\frac{k r}{\nu}\right)^{2}\right]^{1 / 2}} \\
& +\frac{1}{16 \nu^{3}} \int_{0}^{\infty} d r \frac{r V(r)}{\left[1+\left(\frac{k r}{\nu}\right)^{2}\right]^{3 / 2}}\left[1-\frac{6}{\left[1+\left(\frac{k r}{\nu}\right)^{2}\right]}+\frac{5}{\left[1+\left(\frac{k r}{\nu}\right)^{2}\right]^{2}}\right] \\
& -\frac{1}{8 \nu^{3}} \int_{0}^{\infty} d r \frac{r^{3} V^{2}(r)}{\left[1+\left(\frac{k r}{\nu}\right)^{2}\right]^{3 / 2}},
\end{aligned}
$$

with $\nu=l+1 / 2$. 


\section{The asymptotic contribution}

First of all the $k$-integrals in (16) can be done explicitly using

$$
\begin{aligned}
& \int_{m}^{\infty} d k\left[k^{2}-m^{2}\right]^{\frac{1}{2}-s} \frac{\partial}{\partial k}\left[1+\left(\frac{k r}{\nu}\right)^{2}\right]^{-\frac{n}{2}} \\
& =-\frac{\Gamma\left(s+\frac{n-1}{2}\right) \Gamma\left(\frac{3}{2}-s\right)}{\Gamma(n / 2)} \frac{\left(\frac{\nu}{m r}\right)^{n} m^{1-2 s}}{\left(1+\left(\frac{\nu}{m r}\right)^{2}\right)^{s+\frac{n-1}{2}}} .
\end{aligned}
$$

This naturally leads to functions of the type

$$
f(s ; a ; b)=\sum_{\nu=1 / 2,3 / 2, \ldots}^{\infty} \nu^{a}\left(1+\left(\frac{\nu}{m r}\right)^{2}\right)^{-s-b}
$$

which we need around $s=-1 / 2$. The way to deal with this functions has been developed in [18 and for completeness the relevant results are provided in the Appendix, (31). Using these, it is a simple exercise to find

$$
\begin{aligned}
& E_{a s}^{r e n}[\Phi]=-\frac{1}{8 \pi} \int_{0}^{\infty} d r r^{2} V^{2}(r) \ln (m r) \\
& -\frac{1}{2 \pi} \int_{0}^{\infty} d r V(r) \int_{0}^{\infty} d \nu \frac{\nu}{1+e^{2 \pi \nu}} \ln \left|\nu^{2}-(m r)^{2}\right| \\
& -\frac{1}{8 \pi} \int_{0}^{\infty} d r\left[r^{2} V^{2}(r)-\frac{1}{2} V(r)\right] \int_{0}^{\infty} d \nu\left(\frac{d}{d \nu} \frac{1}{1+e^{2 \pi \nu}}\right) \ln \left|\nu^{2}-(m r)^{2}\right| \\
& -\frac{1}{8 \pi} \int_{0}^{\infty} d r V(r) \int_{0}^{\infty} d \nu\left[\frac{d}{d \nu}\left(\frac{1}{\nu} \frac{d}{d \nu} \frac{\nu^{2}}{1+e^{2 \pi \nu}}\right)\right] \ln \left|\nu^{2}-(m r)^{2}\right| \\
& +\frac{1}{48 \pi} \int_{0}^{\infty} d r V(r) \int_{0}^{\infty} d \nu\left[\frac{d}{d \nu}\left(\frac{1}{\nu} \frac{d}{d \nu} \frac{1}{\nu} \frac{d}{d \nu} \frac{\nu^{4}}{1+e^{2 \pi \nu}}\right)\right] \ln \left|\nu^{2}-(m r)^{2}\right|,
\end{aligned}
$$

suitable for numerical evaluation.

\section{Numerical determination of the Jost function and the integration procedure}

Under the assumption that the potential has compact support, let's say $V(r)=0$ for $r \geq R$, the regular solution may be written as

$$
\phi_{l, p}(r)=u_{l, p}(r) \Theta(R-r)+\frac{i}{2}\left[f_{l}(p) \hat{h}_{l}^{-}(p r)-f_{l}^{*}(p) \hat{h}_{l}^{+}(p r)\right] \Theta(r-R) .
$$


This solution and its derivative must be continuous at $r=R$. So the matching conditions are

$$
\begin{aligned}
u_{l, p}(R) & =\frac{i}{2}\left[f_{l}(p) \hat{h}_{l}^{-}(p R)-f_{l}^{*}(p) \hat{h}_{l}^{+}(p R)\right], \\
u_{l, p}^{\prime}(R) & =\frac{i}{2} p\left[f_{l}(p) \hat{h}_{l}^{-1}(p R)-f_{l}^{*}(p) \hat{h}_{l}^{+\prime}(p R)\right] .
\end{aligned}
$$

From these conditions we get the Jost function in terms of the regular solutions,

$$
f_{l}(p)=-\frac{1}{p}\left(p u_{l, p}(R) \hat{h}_{l}^{+\prime}(p R)-u_{l, p}^{\prime}(R) \hat{h}_{l}^{+}(p R)\right),
$$

where we used that the Wronskian determinant of $\hat{h}_{l}^{ \pm}$is $2 i$.

The assumption of the potential $V(r)$ having a finite support is natural within the framework of a numerical calculation. Anyway the potential is assumed to vanish for $r \rightarrow \infty$. So, after some value of the radius it can be viewed as zero within the required precision. This simplifies the numerical procedure considerably. Another situation would occur for a Coulomb like potential which we do not consider in this paper.

In order to determine a unique solution of the differential equation (12), we need to pose the initial value problem corresponding to $\phi_{l, p}(r)$ being the regular solution. We have for $r \rightarrow 0$

$$
\phi_{l, p}(r)=u_{l, p}(r) \sim \hat{j}_{l}(p r) \sim \frac{\sqrt{\pi}}{\Gamma(l+3 / 2)}\left(\frac{z}{2}\right)^{l+1} .
$$

We change to the new function

$$
u_{l, p}(r)=\frac{\sqrt{\pi}}{\Gamma(l+3 / 2)}\left(\frac{p r}{2}\right)^{l+1} g_{l, p}(r)
$$

with the initial value $g_{l, p}(0)=1$. The differential equation for $g_{l, p}(r)$ reads

$$
\left\{\frac{d^{2}}{d r^{2}}+2 \frac{l+1}{r} \frac{d}{d r}-V(r)+p^{2}\right\} g_{l, p}(r)=0,
$$

or, going to the imaginary $p$-axis and writing it as a first order differential equation with $(\partial / \partial r) g_{l, i p}(r)=v_{l, i p}(r)$,

$$
\frac{d}{d r}\left(\begin{array}{c}
g_{l, i p}(r) \\
v_{l, i p}(r)
\end{array}\right)=\left(\begin{array}{cc}
0 & 1 \\
V(r)+p^{2} & -\frac{2}{r}(l+1)
\end{array}\right)\left(\begin{array}{c}
g_{l, i p}(r) \\
v_{l, i p}(r)
\end{array}\right) .
$$

To fix the solution uniquely we only need to fix $v_{l, i p}(0)$. A power series ansatz for $g_{l, i p}(r)$ about $r=0$ shows that for $V(r)=\mathcal{O}\left(r^{-1+\epsilon}\right)$ the condition reads $v_{l, i p}(0)=0$. With this unique solution of (23), the Jost function takes the form

$$
f_{l}(i p)=\frac{2}{\Gamma(l+3 / 2)}\left(\frac{p R}{2}\right)^{l+3 / 2}\left\{g_{l, i p}(R) K_{l+3 / 2}(p R)+\frac{1}{p} g_{l, i p}^{\prime}(R) K_{l+1 / 2}(p R)\right\} .
$$


Finally, doing a partial integration and the substitution $q=\sqrt{k^{2}-m^{2}}$ in Eq. (16), the starting point for the numerical evaluation used for $E_{f}$ is

$$
E_{f}=\frac{1}{\pi} \sum_{l=0}^{\infty}(l+1 / 2) \int_{0}^{\infty} d q\left[\ln f_{l}\left(i \sqrt{q^{2}+m^{2}}\right)-\ln f_{l}^{a s y m}\left(i \sqrt{q^{2}+m^{2}}\right)\right]
$$

where $\ln f_{l}^{\text {asym }}$ is given by Eq. (17).

\section{Examples}

The method described above works for radially symmetric potentials $\Phi(r)$ having compact support $\Phi(r \geq R)=0$. In order to have a finite classical energy (Eq. 9) we also demand $\Phi^{\prime}(r=0)=\Phi^{\prime}(r=R)=0$.

In the following we use dimensionless quantities

$$
\begin{aligned}
\epsilon & =E R, \\
\mu & =m R, \\
\rho & =\frac{r}{R}, \\
V(r) & =\lambda^{\prime} \Phi^{2}(r) \\
& =\frac{1}{R^{2}} v(\rho)=\frac{\lambda^{\prime}}{R^{2}} \varphi^{2}(\rho)
\end{aligned}
$$

such that $\epsilon=\epsilon[\varphi, \mu]$. Therefore, large $\mu$ corresponds to large $R$ and to large $m$, respectively.

We studied two types of potentials. Type A is lump-like concentrated around $r=0$ whereas type $\mathrm{B}$ has its maximum at $R / 2$, acting like a spherical wall:

$$
\begin{aligned}
\varphi_{A}(\rho) & =\frac{a\left(1-\rho^{2}\right)^{2}}{a+\rho^{2}}, \\
\varphi_{B}(\rho) & =\frac{16 a \rho^{2}(1-\rho)^{2}}{a+(1-2 \rho)^{2}} .
\end{aligned}
$$

The parameter $a$ allows to vary the shape of the potentials, as indicated in the insets of the figures.

Our numerical implementation of Eq. (25) showed that the sum over angular momenta converges quite fast, usually after 10-50 terms. Hereby, the numerical $q$-integration procedure has to solve the system of ordinary differential equations Eq. (23) for every evaluation of the Jost function $f_{l}(i p)$.

As a check, we compared our results with the asymptotic formulae

$$
\epsilon \sim \begin{cases}\frac{1}{16 \pi^{2}} A_{2} \log \frac{1}{\mu} & \text { for } \mu \rightarrow 0 \\ \frac{-1}{32 \pi^{2}} \frac{A_{3}}{\mu^{2}} & \text { for } \mu \rightarrow \infty\end{cases}
$$


where $A_{2}=\frac{1}{2} \int \mathrm{d} \vec{x} V(r)^{2}$ is the second heat kernel coefficient and $\left.A_{3}=\frac{1}{6} \int \mathrm{d} \vec{x}\left(\frac{1}{2} V(r) \Delta V(r)-V(r)^{3}\right)\right)$ is the third one. These relations follow from scaling arguments together with the well known property of the heat kernel expansion being the asymptotic expansion for large mass.

The most determining property of the potential $V(r)$ in the attractive case $\lambda^{\prime}<0$ is the number and depth of bound states. The parameter dependence of the $l=0$ bound states, determined as zeroes of the $l=0$ Jost function, Eq. (24), is shown in figs. 1 and 2. Number as well as depth of the bound states increases with increasing $\left|\lambda^{\prime}\right|$ and with increasing $a$, too.

As Eq. (25) shows, the Casimir energy develops an imaginary part (corresponding to particle creation by the external field) when the $q$-integration passes a zero of the Jost function. $E_{f}$ is real as long as $\mu$ is larger than the depth of the highest bound state. Therefore we indicate in our figures $3 \sqrt{6}$ the positions of bound states on the $\mu$ axis by vertical lines where the plots of the Casimir energy end.

To show clearly the influence of the form and spectrum of $V$ on the vacuum energy, we consider the dependence of the energy for fixed $\lambda^{\prime}$ and different values of the parameter $a$, figs. 3 and 4 , as well as for fixed shape $a$ and different depths $\lambda^{\prime}$ in figs. 5 and 6 .

These figures demonstrate that the vacuum energy is determined by two competing contributions, the positive contribution of the scattering states and the negative contribution of the bound states of the potential.

Let us start with a description and interpretation of figs. 3 and 4 . For $a$ large, the contribution of the bound state(s) to the vacuum energy is large enough to overcompensate the contribution of the scattering states and the energy is negative. At some "critical value" of $a$, the energy of the only remaining bound state is so small that the positive contributions of the scattering states get the upper hand, and the vacuum energy is positive. Finally, for $a \rightarrow 0$ we have normalized $E_{\varphi}^{r e n}[\Phi=0]=0$, such that at some point $E_{\varphi}^{r e n}$ starts to decrease again with decreasing $a$.

The same features are clearly recovered in figs. 5 and 6. For large enough $\left(-\lambda^{\prime}\right)$ a negative vacuum energy energy is obtained. With decreasing $\left(-\lambda^{\prime}\right)$ the energy increases and can be positive or negative depending on the parameter $\mu$. Again, at some point the scattering states dominate, the energy gets positive and tends to zero as $\lambda^{\prime}$ tends to zero. For positive $\lambda^{\prime}$ the energy starts to increase again, being virtually identical for the two values $\lambda^{\prime}= \pm 2$, and it seems that $E_{\varphi}^{r e n}$ is a monotonically increasing function of $\lambda^{\prime}$ for $\lambda^{\prime} \geq 0$. Since we see no qualitative difference between our two potential types $\mathrm{A}$ and $\mathrm{B}$, we expect these features to be universal.

Given that the dependence of the energy on $\lambda^{\prime}$ is somewhat nontrivial, let us show this dependence explicitely for type A potentials in fig. (7).

As long as no bound states exist, the sign of $\lambda^{\prime}$ is not important and the energy is nearly symmetric about $\lambda^{\prime}=0$. The contribution to the energy of 
the scattering states does not depend much on the sign of $\lambda^{\prime}$. The symmetry is disturbed as soon as a bound state shows up and the energy then decreases with decreasing $\lambda^{\prime}$ because the bound state depth as well as the number of bound states increases.

\section{Conclusions}

We demonstrated that our method is quite feasible for calculations of the Casimir energy in realistic 3-dimensional examples for arbitrary spherical symmetric external potentials as long as they are smooth and have compact support.

The behavior of the Casimir energy shows some interesting features. The existence of bound states is a necessary but by no means sufficient condition for a negative vacuum energy. Even in the presence of bound states the sign of the energy can depend on the scale parameter $\mu$, i.e the mass of the quantum field measured in units of the size $R$ of the potential.

For shallow potentials without bound states (small $\left|\lambda^{\prime}\right|$ ) the Casimir energy is nearly invariant under a change of the sign of the potential whereas for deep attractive potentials the negative contribution of the bound states dominates the vacuum energy.

On the other hand we found no qualitative differences between a lump-like and a ring-like classical background configuration.

The method developed here can be used for the calculation of quantum corrections to classical objects like Skyrmions and others.

KK had been supported by DFG under grant number Bo 1112/4-2.

\section{Appendix: Analytical continuation of the asymp- totic contributions}

The essential formulas for the basic series $f(s ; a ; b)$, Eq. (19), in the calculation are the following (we use $x=m r$ ):

$$
\begin{aligned}
\sum_{\nu=1 / 2,3 / 2, \ldots}^{\infty} \nu^{2 n+1}\left(1+\left(\frac{\nu}{x}\right)^{2}\right)^{-s} & =\frac{1}{2} \frac{n ! \Gamma(s-n-1)}{\Gamma(s)} x^{2 n+2} \\
& +(-1)^{n} 2 \int_{0}^{x} d \nu \frac{\nu^{2 n+1}}{1+e^{2 \pi \nu}}\left(1-\left(\frac{\nu}{x}\right)^{2}\right)^{-s} \\
& +(-1)^{n} 2 \cos (\pi s) \int_{x}^{\infty} d \nu \frac{\nu^{2 n+1}}{1+e^{2 \pi \nu}}\left(\left(\frac{\nu}{x}\right)^{2}-1\right)^{-s}
\end{aligned}
$$




$$
\begin{array}{r}
\sum_{\nu=1 / 2,3 / 2, \ldots}^{\infty} \nu^{2 n}\left(1+\left(\frac{\nu}{x}\right)^{2}\right)^{-s}=\frac{1}{2} \frac{\Gamma(n+1 / 2) \Gamma(s-n-1 / 2)}{\Gamma(s)} x^{2 n+1} \\
-(-1)^{n} 2 \sin (\pi s) \int_{x}^{\infty} d \nu \frac{\nu^{2 n}}{1+e^{2 \pi \nu}}\left(\left(\frac{\nu}{x}\right)^{2}-1\right)^{-s}
\end{array}
$$

Using partial integrations one can get representations valid for all values of $s$ needed. The explicit results applied for the calculation of (16) are [we shall use the notation $f(a ; b)=f(-1 / 2 ; a ; b)]$,

$$
\begin{aligned}
f(1 ; 1 / 2)= & -\frac{1}{2} x^{2}+\frac{1}{24} \\
\left.\frac{d}{d s}\right|_{s=-1 / 2} f(s ; 1 ; 1 / 2)= & -\frac{1}{2} x^{2}-2 \int_{0}^{\infty} d \nu \frac{\nu}{1+e^{2 \pi \nu}} \ln \left|1-\left(\frac{\nu}{x}\right)^{2}\right| \\
f(1 ; 3 / 2)= & \frac{x^{2}}{2(s+1 / 2)}+x^{2} \ln x \\
& +x^{2} \int_{0}^{\infty} d \nu\left(\frac{d}{d \nu} \frac{1}{1+e^{2 \pi \nu}}\right) \ln \left|\nu^{2}-x^{2}\right|, \\
f(3 ; 5 / 2)= & \frac{x^{4}}{2(s+1 / 2)}+(\ln x-1 / 2) x^{4} \\
& +\frac{x^{4}}{2} \int_{0}^{\infty} d \nu\left[\frac{d}{d \nu}\left(\frac{1}{\nu} \frac{d}{d \nu} \frac{\nu^{2}}{1+e^{2 \pi \nu}}\right)\right] \ln \left|\nu^{2}-x^{2}\right|, \\
f(5 ; 7 / 2)= & \frac{x^{6}}{2(s+1 / 2)}+(\ln x-3 / 4) x^{6} \\
& +\frac{x^{6}}{8} \int_{0}^{\infty} d \nu\left[\frac{d}{d \nu}\left(\frac{1}{\nu} \frac{d}{d \nu} \frac{1}{\nu} \frac{d}{d \nu} \frac{\nu^{4}}{1+e^{2 \pi \nu}}\right)\right] \ln \left|\nu^{2}-x^{2}\right|,
\end{aligned}
$$

which provide the needed expansions about $s=0$ in (16).

\section{References}

[1] N.D. Birrell and P.C.W. Davie. Quantum Fields in Curved Space. Cambridge University Press, Cambridge, 1982.

[2] G. t'Hooft. Nucl. Phys., B79:276, 1974.

[3] A. M. Polyakov. Particle spectrum in the quantum field theory. JETP Lett., 20:194-195, 1974.

[4] F. R. Klinkhamer and N. S. Manton. A saddle point solution in the weinbergsalam theory. Phys. Rev., D30:2212, 1984. 
[5] T. H. R. Skyrme. A unified field theory of mesons and baryons. Nucl. Phys., 31:556, 1962.

[6] E. Elizalde, S.D. Odintsov, A. Romeo, A.A. Bytsenko, and S. Zerbini. Zeta Regularization Techniques with Applications. World Scientific, Singapore, 1994.

[7] A. A. Actor and I. Bender. Casimir effect for soft boundaries. Phys. Rev., D52:3581, 1995.

[8] M. Bordag, D. Hennig, and D. Robaschik. Vacuum energy in quantum field theory with external potentials concentrated on planes. J. Phys. A, A25:4483, 1992.

[9] Joon-Il Kim and Byung-Yoon Park. Casimir energy of the skyrmion due to kaon vacuum fluctuations. Phys. Rev., D57:2853-2858, 1998.

[10] Norberto N. Scoccola and Hans Walliser. Casimir corrections in the bound state soliton model. Phys. Rev., D58:094037, 1998.

[11] M. Bordag. Vacuum energy in smooth background fields. J. Phys., A28:755766, 1995.

[12] M. Bordag and K. Kirsten. Vacuum energy in a spherically symmetric background field. Phys. Rev., D53:5753-5760, 1996.

[13] J. Baacke. Numerical evaluation of the one loop effective action in static backgrounds with spherical symmetry. Z. Phys., C47:263-268, 1990.

[14] E. Farhi, N. Graham, P. Haagensen, and R. L. Jaffe. Finite quantum fluctuations about static field configurations. Phys. Lett., B427:334, 1998.

[15] M. Bordag, K. Kirsten, and D. Vassilevich. On the ground state energy for a penetrable sphere and for a dielectric ball. Phys. Rev., D59:085011, 1999.

[16] Steve Blau, Matt Visser, and Andreas Wipf. Zeta functions and the casimir energy. Nucl. Phys., B310:163, 1988.

[17] J.R. Taylor. Scattering Theory. Wiley, New York, 1972.

[18] E. Elizalde, M. Bordag, and K. Kirsten. Casimir energy for a massive fermionic quantum field with a spherical boundary. J. Phys. A, A31:1743, 1998. 


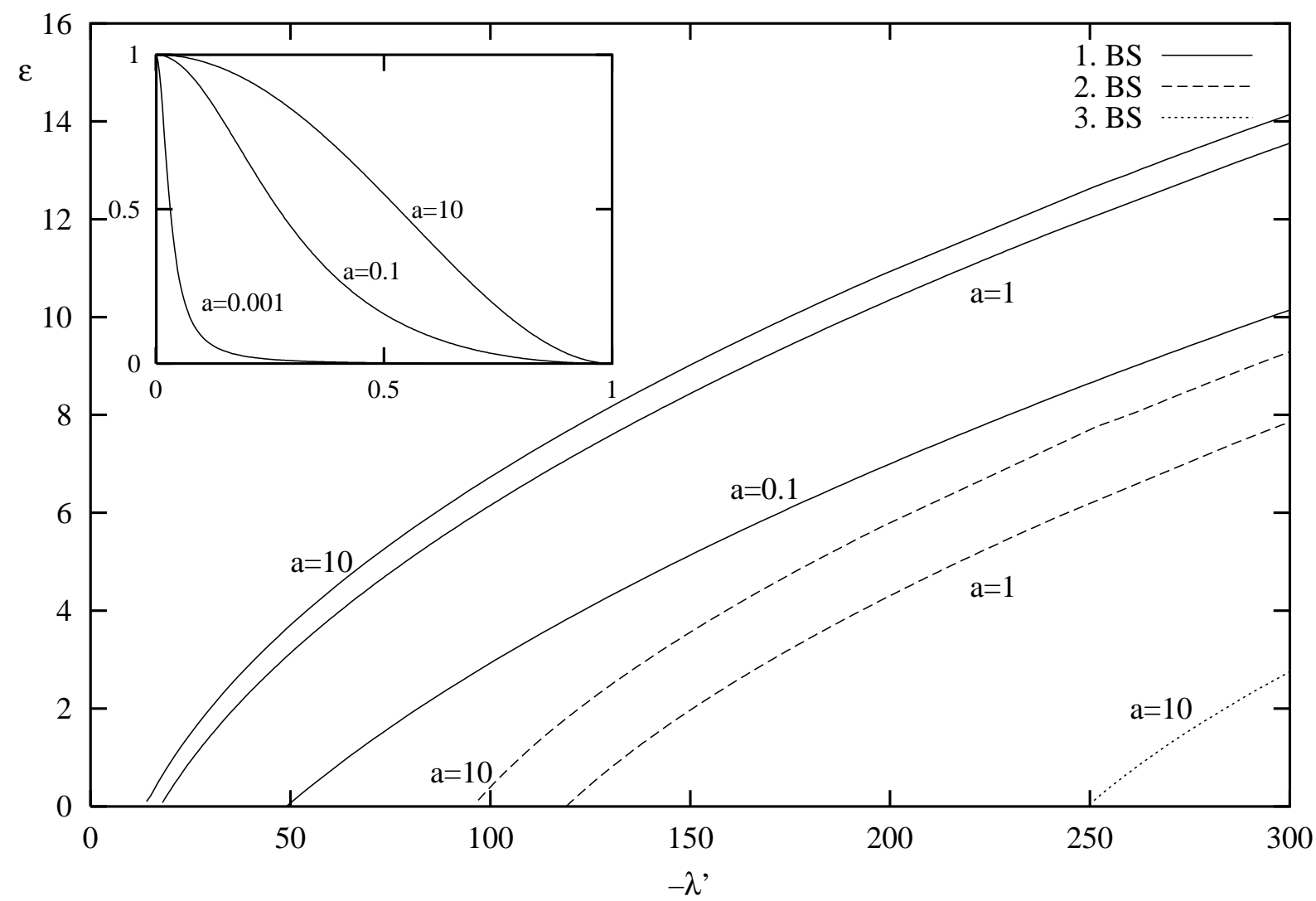

Figure 1: Energy of bound states for type A potential and negative $\lambda^{\prime}$. (The inset shows $\varphi(\rho)$ where $\left.V(\rho)=\lambda^{\prime} \varphi^{2}(\rho)\right)$ 


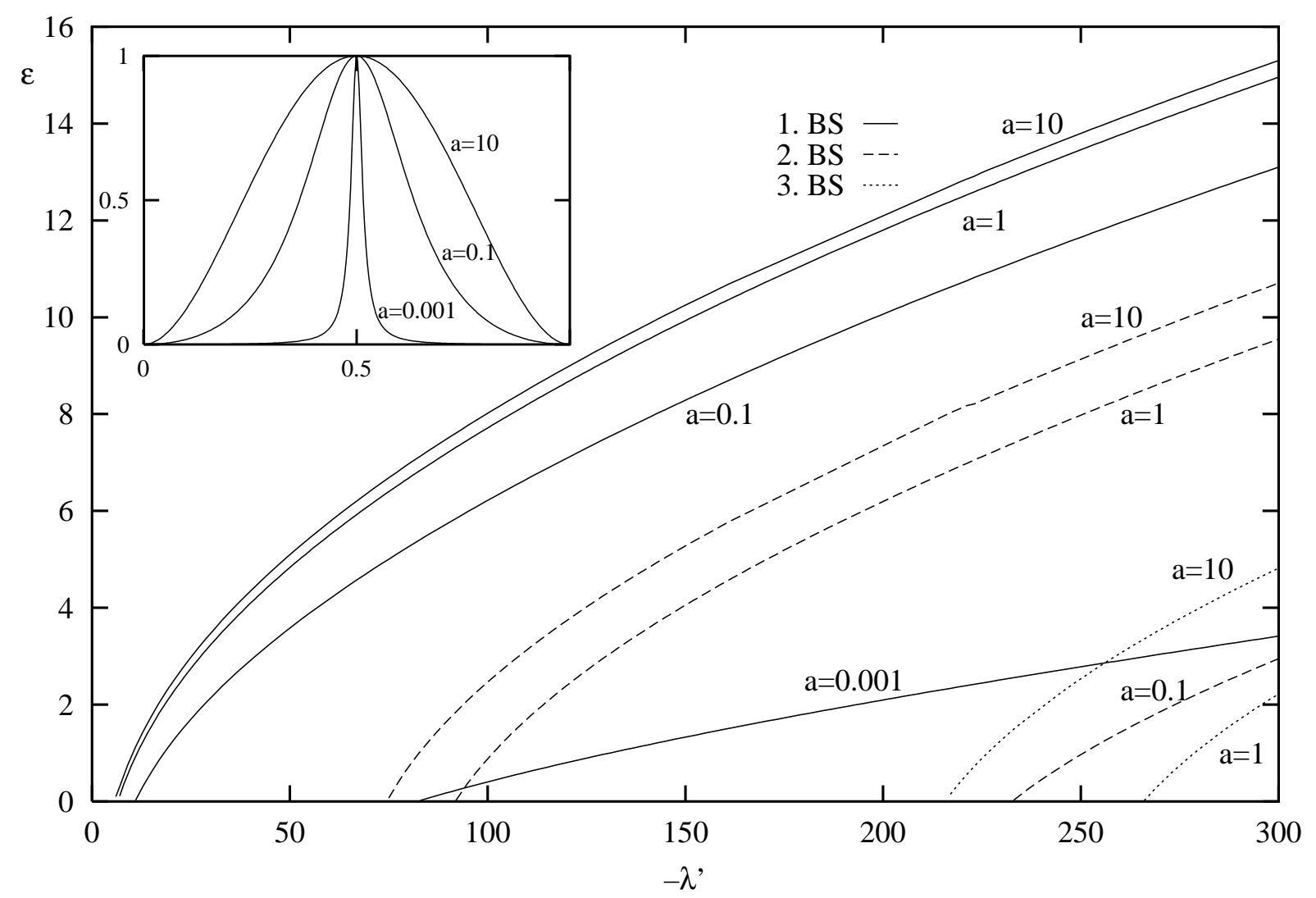

Figure 2: Energy of bound states for type B potential and negative $\lambda^{\prime}$. (The inset shows $\varphi(\rho)$ where $\left.V(\rho)=\lambda^{\prime} \varphi^{2}(\rho)\right)$ 


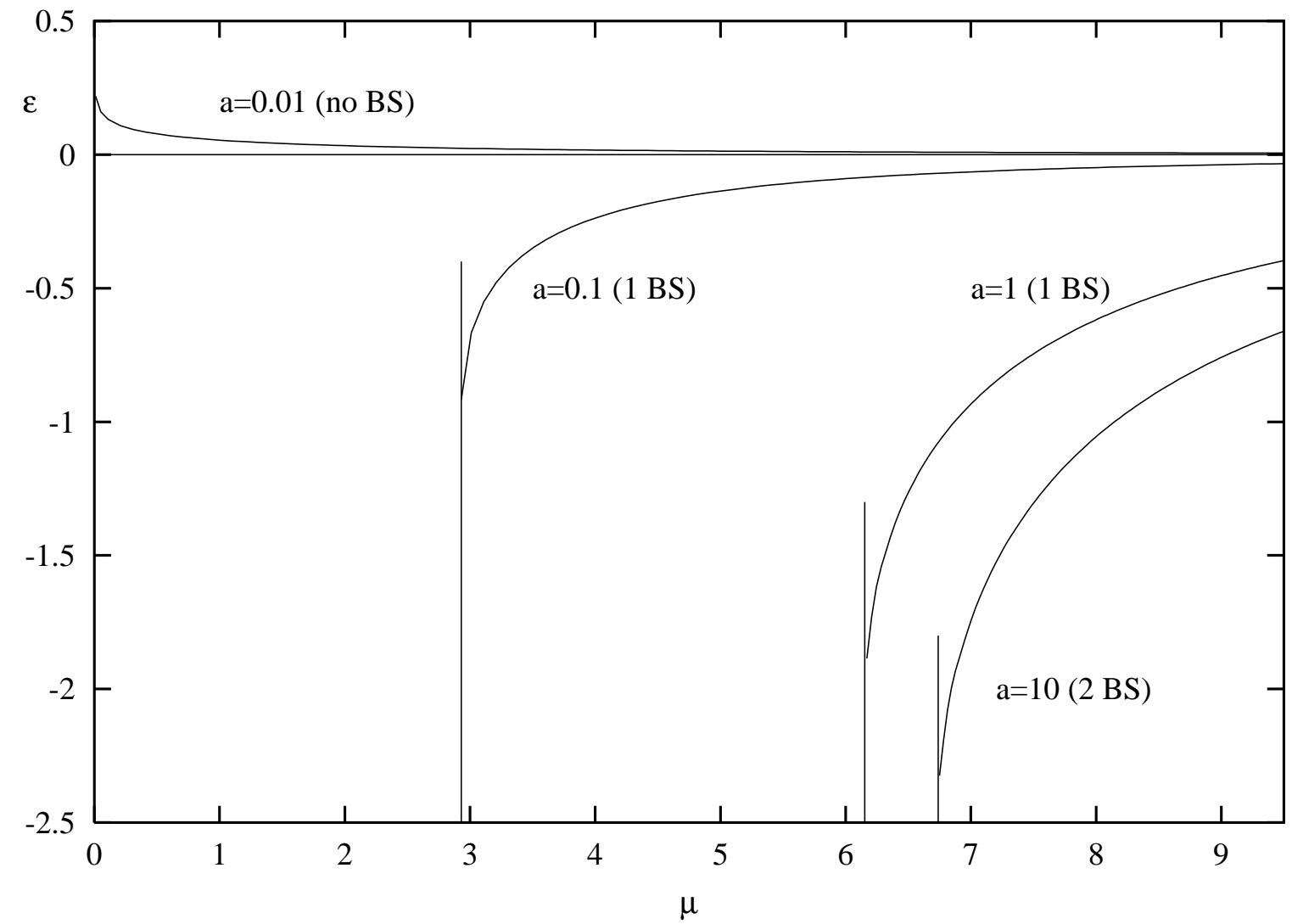

Figure 3: Energy for type A potentials of different shapes with $\lambda^{\prime}=-100$. The positions of bound states (BS) at the $\mu$ axis are shown as vertical lines. 


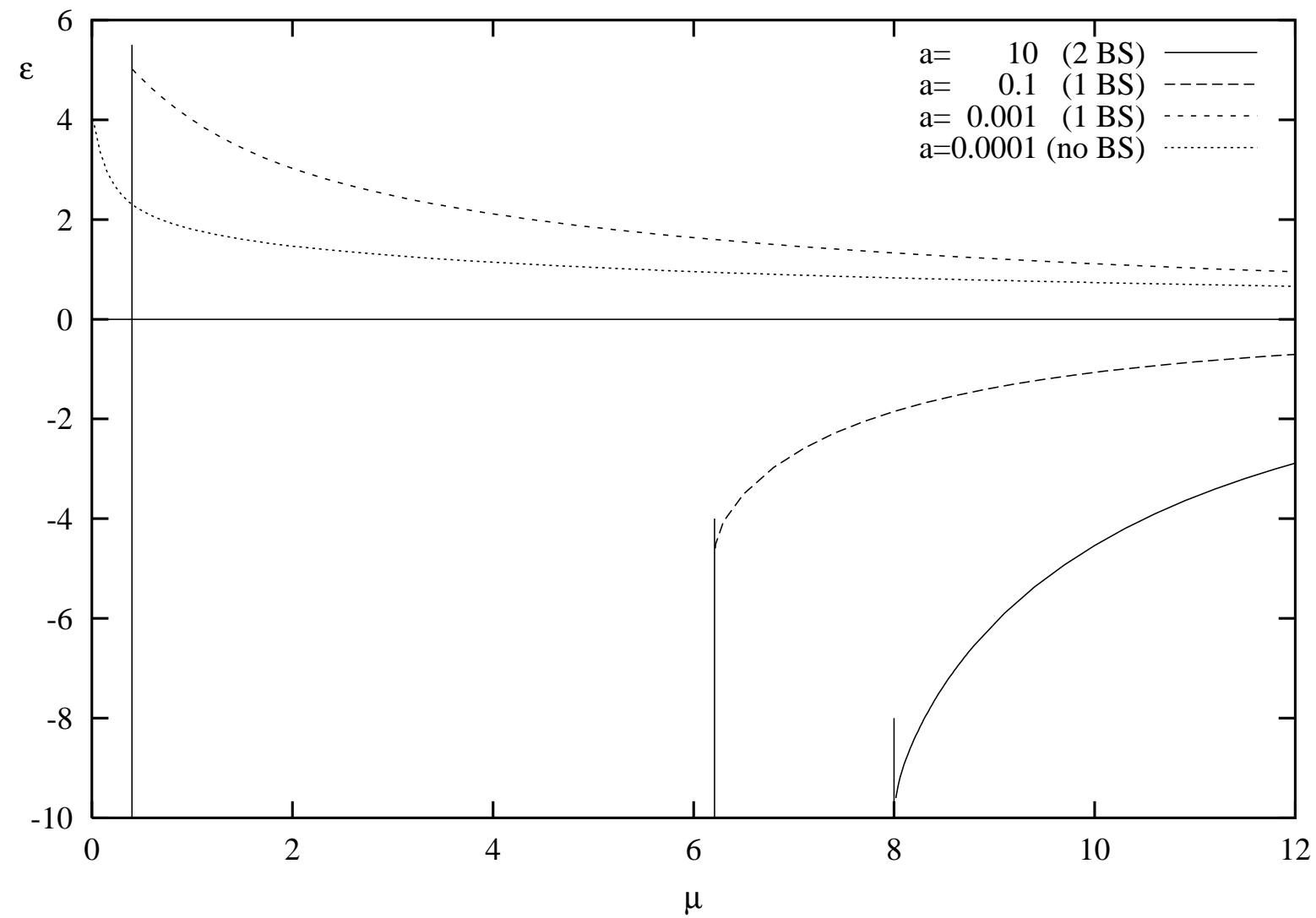

Figure 4: Energy for type B potentials of different shapes with $\lambda^{\prime}=-100$. The positions of bound states (BS) at the $\mu$ axis are shown as vertical lines. 


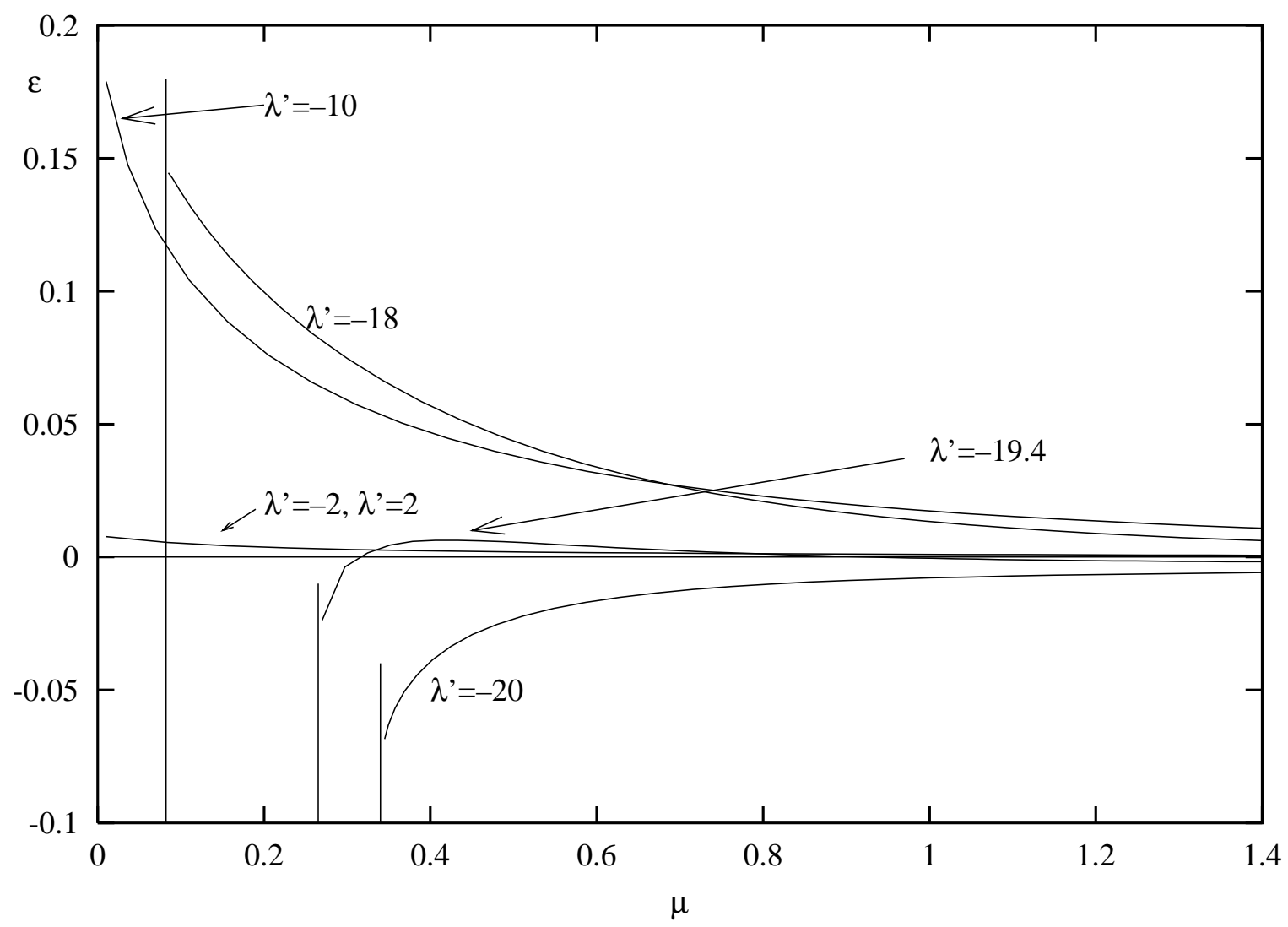

Figure 5: Energy for type A potentials of different magnitudes $\lambda^{\prime}$ with equal shape parameter $a=1$. The positions of bound states existing for $\lambda^{\prime}=-18,-19.4$ and -20 at the $\mu$ axis are shown as vertical lines. 


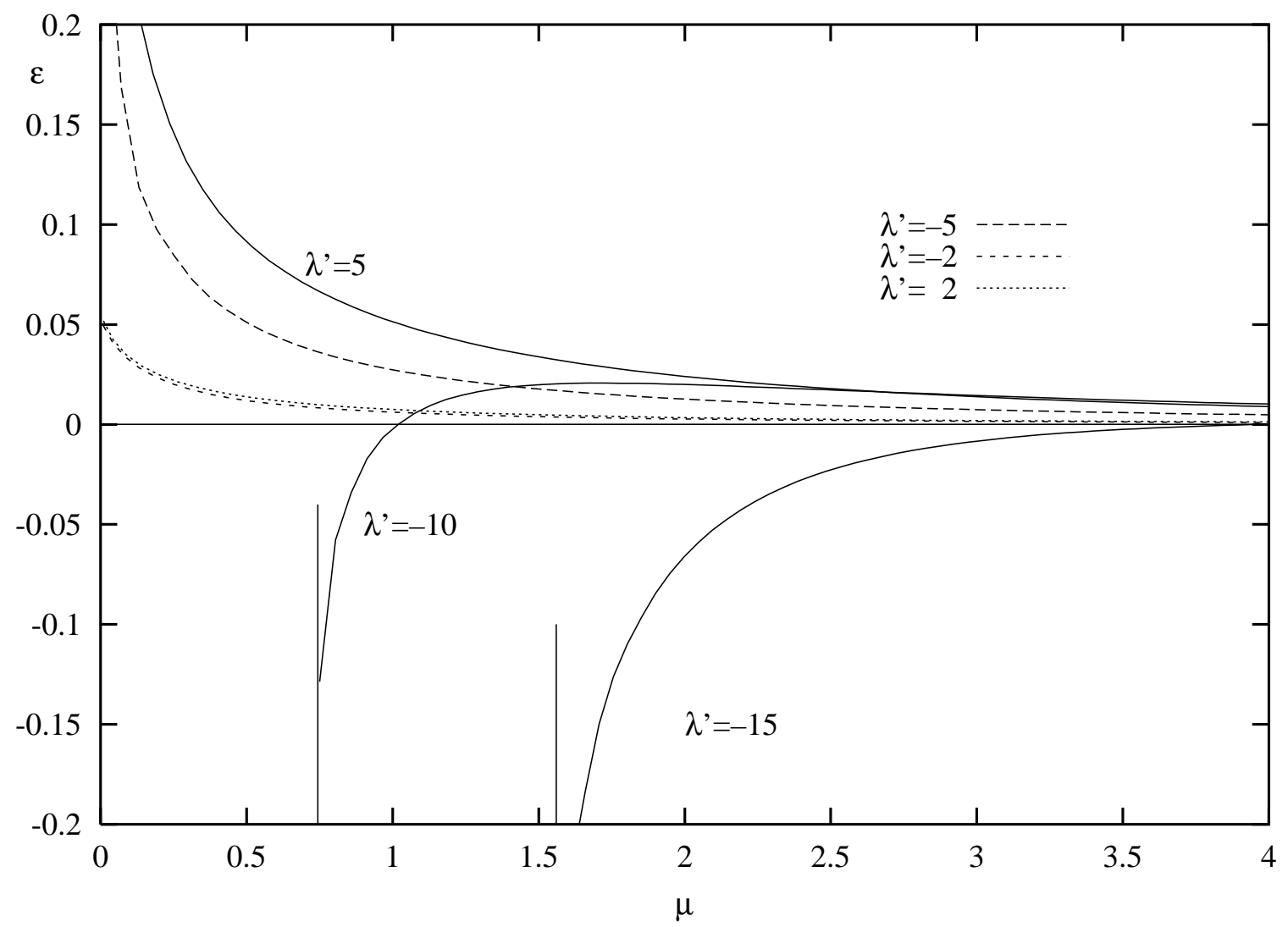

Figure 6: Energy for type B potentials of different magnitudes $\lambda^{\prime}$ with equal shape parameter $a=1$. The positions of bound states existing for $\lambda^{\prime}=-15$ and -10 at the $\mu$ axis are shown as vertical lines. 


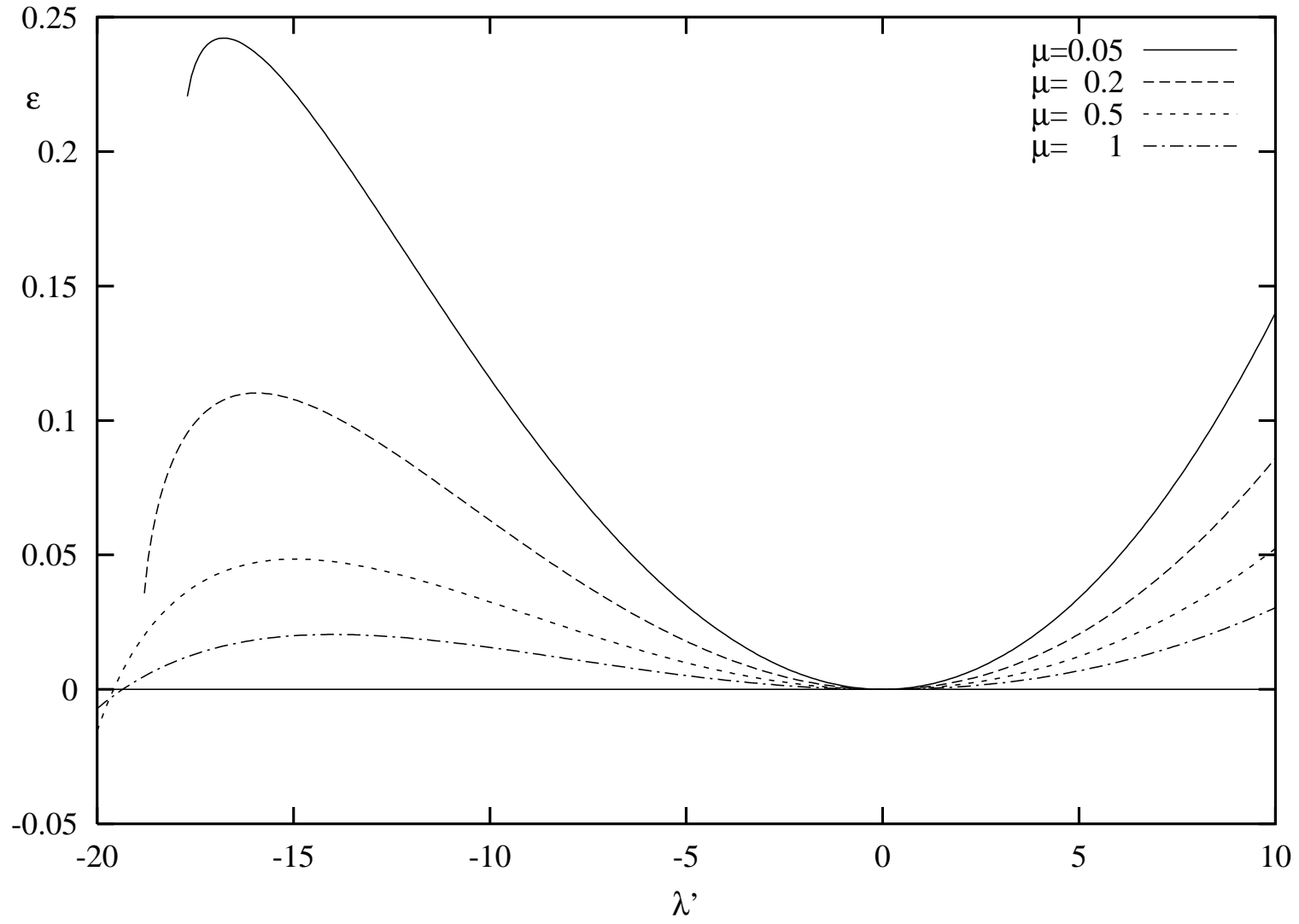

Figure 7: Energy for type A potentials for different magnitudes $\lambda^{\prime}$ and equal shape parameter $a=1$ as a function of the parameter $\mu$. 